\title{
Regulatory pathways for smart grid development in China
}

6.1 Government targets for China's future electric power system - 120

6.2 Underlying scenarios - 122

6.3 Drawing the roadmaps - 122

6.3.1 Reliability/Security of Supply scenario - 123

6.3.2 Ecological Sustainability scenario - 128

6.3.3 Affordability/Competition scenario - 132

6.4 Discussion of the three roadmaps - 136

References - 138 
Chapter at a glance

- There are inherent conflicts between the fundamental energy policy goals of reliability, affordability, and sustainability. Government priorities on the energy policy goals are often reflected in energy sector regulation.

- Seven recommendations have been presented in support of smart grid development in China. In principle, each recommendation is intended as support for the fundamental energy policy goals. Nevertheless, the implementation sequence of the recommendations is not arbitrary with respect to the energy policy goals.

- In order to give policy makers an impression of how policy goal prioritization influences the timeline in which the recommendations should be implemented, the present chapter will outline three possible regulatory pathways. Each of these pathways prioritizes one specific goal of the energy policy triangle and develops a suitable implementation roadmap. These roadmaps are intended to serve as blueprints for policy makers, who have to decide about proper regulation based on the individual Chinese prioritization of energy policy goals.

\subsection{Government targets for China's future electric power system}

The following chapter will present the main targets of China's government for each stage of the electric power system. These targets are the basis for a comprehensive description of the scenarios underlying the regulatory pathways. The targets are based on the following documents:

- $12^{\text {th }}$ Five-Year Plan for Energy Development, issued by China's State Council in 2013 [1],

- Opinions on Accelerating the Development of Environmental Industry, issued by China's State Council in 2013 [2],

- Key Information at a glance - China's $12^{\text {th }}$ FiveYear Plan for Renewable Energy Development, issued by NEA in 2012 [3],

- China's 2012 Energy Policy, issued by the Information Office of the State Council in 2012 [4].

- Understanding China's $12^{\text {th }}$ Five-Year Energy Plan, issued by IEA in 2013 [5].
Targets for the power generation sector In order to cope with rapidly increasing power consumption and to ensure power system reliability, China’s government plans to increase total electricity generation capacities during the next years from $970 \mathrm{GW}$ in 2010 to $1490 \mathrm{GW}$ in 2015 . It is projected that generation capacities will increase further to $1935 \mathrm{GW}$ in 2020 [6].

More specifically, China's government aims at vigorously developing distributed and renewable energy sources. From 880 TWh in 2011, electricity generated solely from renewable sources is projected to increase to more than $1,200 \mathrm{TWh}$ in 2015 . The projected increases in generation capacities for solar power, wind power, and biomass power are impressive. If these expansion targets are to be achieved, RES must be integrated in the grid more effectively than today. The Chinese government additionally aims at stopping the deterioration of air quality as well as the increase of $\mathrm{CO}_{2}$ emissions. The target is therefore for the total amount of discharge of $\mathrm{SO}_{2}$ and $\mathrm{NO}_{\mathrm{x}}$ to remain constant between 2015 and 2020. Also, the increase in $\mathrm{CO}_{2}$ emissions will be slowed in spite of the planned coal power generation growth.

Government targets for the power generation sector at a glance

- Roughly double generation capacities from 2010 to 2020

- Vigorously develop RES

- Stop the deterioration of air quality.

- Reduce the growth of $\mathrm{CO}_{2}$ emissions

Targets for the power logistics sector China's government plans to significantly expand transmission and distribution grids. The capacity of China's transmission grids is planned to double by 2020 (compared to 2010), creating a national backbone grid, interconnecting different Chinese regions and improving electricity transmission from the energy bases to the load centers. The plan also include upgrading urban and rural distribution grids, promoting distribution intelligence, and improving the capacity and reliability of the overall power supply. This enhancement of distribution grids primarily 
aims at reducing blackout times by roughly $40 \%$ from 2012 to 2020 . The government also aims at improving asset utilization of the grid infrastructure by peak shaving and by reducing the line loss rate from $6.5 \%$ in 2010 to $6.3 \%$ in 2015 .

With the goal of facilitating the integration of large-scale and intermittent RES, the Chinese government aims at promoting energy storage technologies by funding R\&D on energy storage, energy conversion and related key technologies.

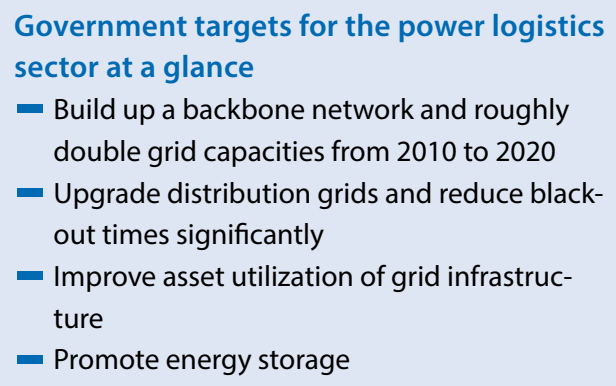

Targets for the power trade and retail sector The Chinese government plans to establish a more market-oriented energy system to increase competition and affordability. Administrative reforms of SOE are planned to take place: transmission and distribution will be separated in a number of pilot projects and independent power trading institutions will be established. Electricity markets and price-based coordination mechanisms are planned to be introduced: the government strives to form a pricing mechanism where markets set on-grid and off-grid prices and the government only sets the price for electricity transmission and distribution (network charge), the economic dispatch for power generation will be increased, and time-of-use prices, seasonal tariffs and interruptible load electricity tariffs are planned for more widespread introduction.

Government targets for the power trade and retail sector at a glance

- Establish a modern energy market system

- Promote reforms in key energy sectors

- Improve energy pricing mechanisms
Targets for the power consumption sector One of the government's major targets is to increase the efficiency of power use in order to reduce growth in total electricity consumption. Specifically, the government intends to reduce energy consumption per unit of GDP by roughly $16 \%$ from 2010 to 2015 . Given that some of China's most remote regions are still not connected to the electric power grid, China's government also wants to provide universal access to electric power even in most remote regions of China by 2015 .

\section{Government targets for the power consumption sector at a glance \\ - Increase efficiency of power use \\ - Provide universal access to electric power even in most remote regions of China by 2015}

Cross-sector targets With regard to power system governance, the government aims at improving the planning and implementation of power sector development: targets and responsibilities of different governmental institutions will be clarified and coordinated; local governments and the pertinent departments of the State Council will be made responsible for binding indicators such as the share of non-fossil fuels in energy consumption or the intensity of energy consumption.

Simultaneously the government is moving forward to strengthen energy industry management by accelerating the introduction of an Energy Law and by enacting amendments to the Coal Law and the Electric Power Law. Also, technology and equipment in the energy sector will be further standardized and capacities of statistics, monitoring, prediction, and warning will be built up.

The Chinese government envisages rationalizing energy investment and the management system of SOE. The government wants to explicitly adhere to the dominance of its state-owned economy in key energy sectors related to national security and economy but, at the same time, it wants to improve the management and assessment mechanisms of SOE.

Regardless of the adherence to the dominant position of SOE in China's power system, the gov- 
ernment also has the objective of diversifying investments in the energy sector. Approval for energy infrastructure investments is intended to become less restrictive so that the participation of private and foreign capital is encouraged. Single energy markets, like the oil market, will be opened up to competition. The government wants to strengthen the innovation potential of China's environmental industry in order to enable the industry to increase the supply of green products, expand market demand, and enhance export prospects.

Another goal is to accelerate the development of the environmental industry in order to promote the development of technologies and equipment that save energy, protect the environment, and encourage reuse of resources.

\section{Governmental cross-sector targets}

at a glance

- Improve planning and implementation

- Strengthen the management of the energy industry

- Rationalize energy investment and the management system of SOE

- Diversify energy investment

- Accelerate the development of the environmental industry

\subsection{Underlying scenarios}

In the following chapter, three smart grid development scenarios will be presented, based on different energy policy goals. For each scenario, the recommendations made in $>$ Chap. 5 will be prioritized and their interdependencies analyzed. The results of this analysis are scenario-based propositions for the implementation sequences of the recommendations. They will be presented in the form of a roadmap. Each roadmap is intended to support China's smart grid development with a focus on one particular scenario. The smart grid scenarios presented in the chapter are:

- Reliability/Security of Supply: In this scenario, the goal is to maximize electricity availability and to minimize outages and disruptions of the electricity supply.
- Ecological Sustainability: The second scenario focuses on improving air quality as well as on decreasing and potentially stopping the growth of $\mathrm{CO}_{2}$ emissions in order to contribute to climate protection. Amongst others, this is achieved by an accelerated installation of RES capacities.

- Affordability/Competition: Competition is a key factor for innovation and affordability. This scenario will consequently focus on the creation of level playing fields and of incentives for market participation of new market actors through appropriate market design.

The government targets presented in $>$ Sect. 6.1 are associated to the three scenarios (see - Fig. 6.1). In some cases, a specific target contributes to more than one energy policy goal. Yet, in these cases, in an effort to increase clarity, only the most obvious association to one specific policy goal will be presented. Two targets are general smart grid development targets as recorded in the $12^{\text {th }}$ Five-Year Plan for Energy Development. They are placed in the center of the triangle, as smart grids are seen as a means to contribute to all energy policy goals.

\subsection{Drawing the roadmaps}

For migrating from today's electric power system towards smart grids based on one of the given scenarios, it is favorable to implement the recommendations in a specific timeline. For deriving the implementation sequences, the recommendations were sorted by priority in a first step. In a second step, main aspects of the recommendations in each scenario were collected and allocated to the timeframes short term (2015-2016), medium term (2017-2020) and long term (2021-2030). Interdependencies between main aspects were explored additionally. Afterwards the recommendations were arranged chronologically.

The following three sections show recommended priorities, their main aspects, interdependencies, and the sequences worked out for the implementation for each scenario. Recommendations and dependencies are highlighted within the order of their respective importance for the scenario. In 


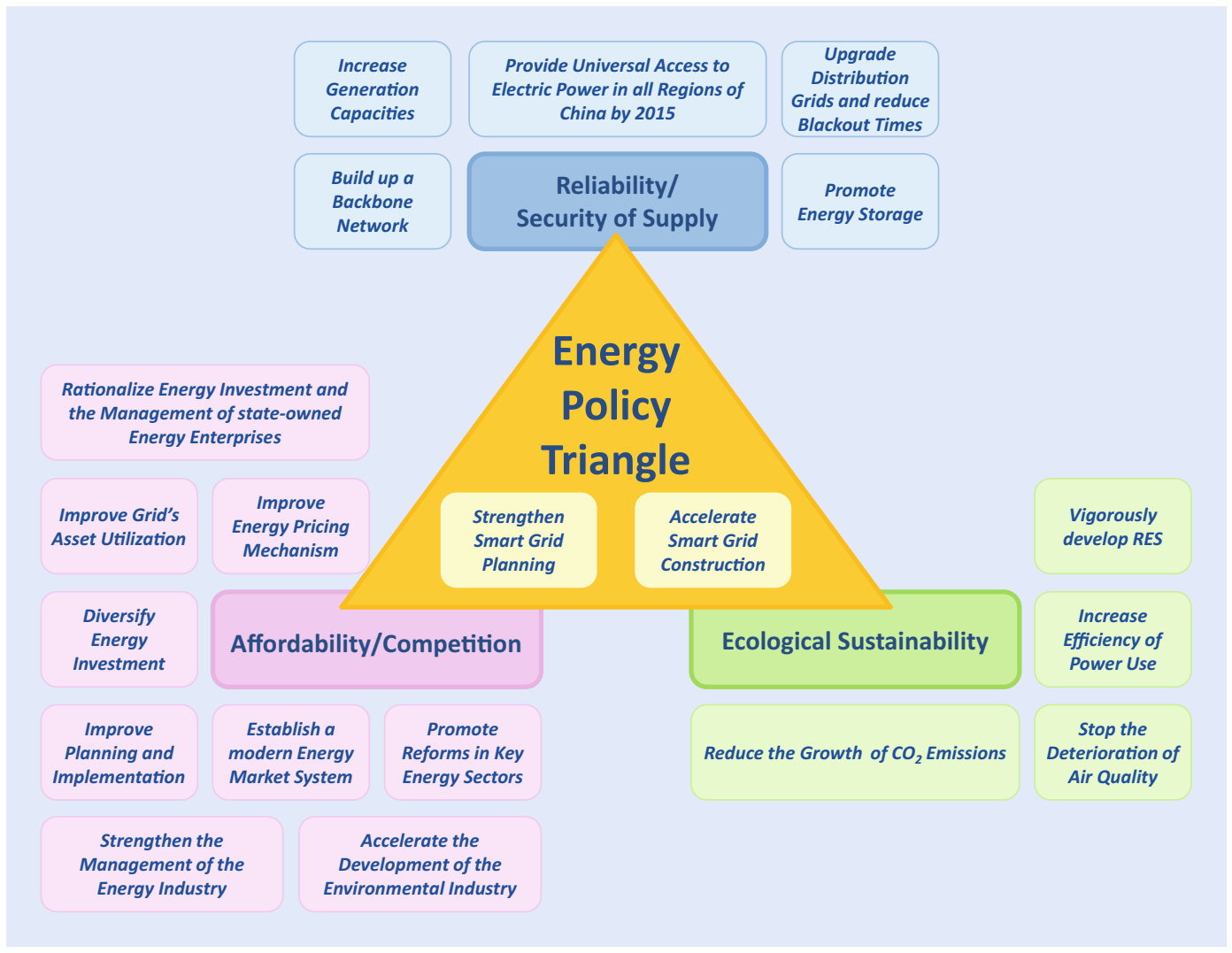

- Fig. 6.1 Scenarios and their associations with energy policy goals

addition, a visualization pertaining to the scenario and summarizing the timeframes of the respective measures of each recommendation is also presented. Furthermore, a possible sequence of starting points for work on the recommendations has been worked out. The justifications for the prioritization of the recommendations are discussed together with each chart. Moreover, the specific measures to be executed for each recommendation, their respective interdependencies, and their timeframes are explained.

\subsubsection{Reliability/Security of Supply scenario}

Looking at the overall prioritization with respect to the Reliability/Security of Supply scenario, it can be seen that the recommendations to Define a longterm strategy for the electricity sector and establish an independent and powerful regulator, Coordinate network expansion planning for electricity grid expansion and upgrade, and to Improve grid integration of $R E S$ have a high priority (• Fig. 6.2). Remember that these priorities do not necessarily correspond to the proposed implementation sequence, because interdependencies within the given scenario have to be analyzed before recommendations are implemented.

Define a long-term strategy for the electricity sector and establish an independent and powerful regulator Effective government work is a precondition for the efficient development of smart grids. Therefore, roles and responsibilities for government administration and regulation have to be set from the start. As a consequence, it is important to implement in the short term all the measures proposed in the recommendation to Define a long-term strategy for the electricity sector and establish an independent and powerful regulator: 


\section{Reliability / Security of Supply}

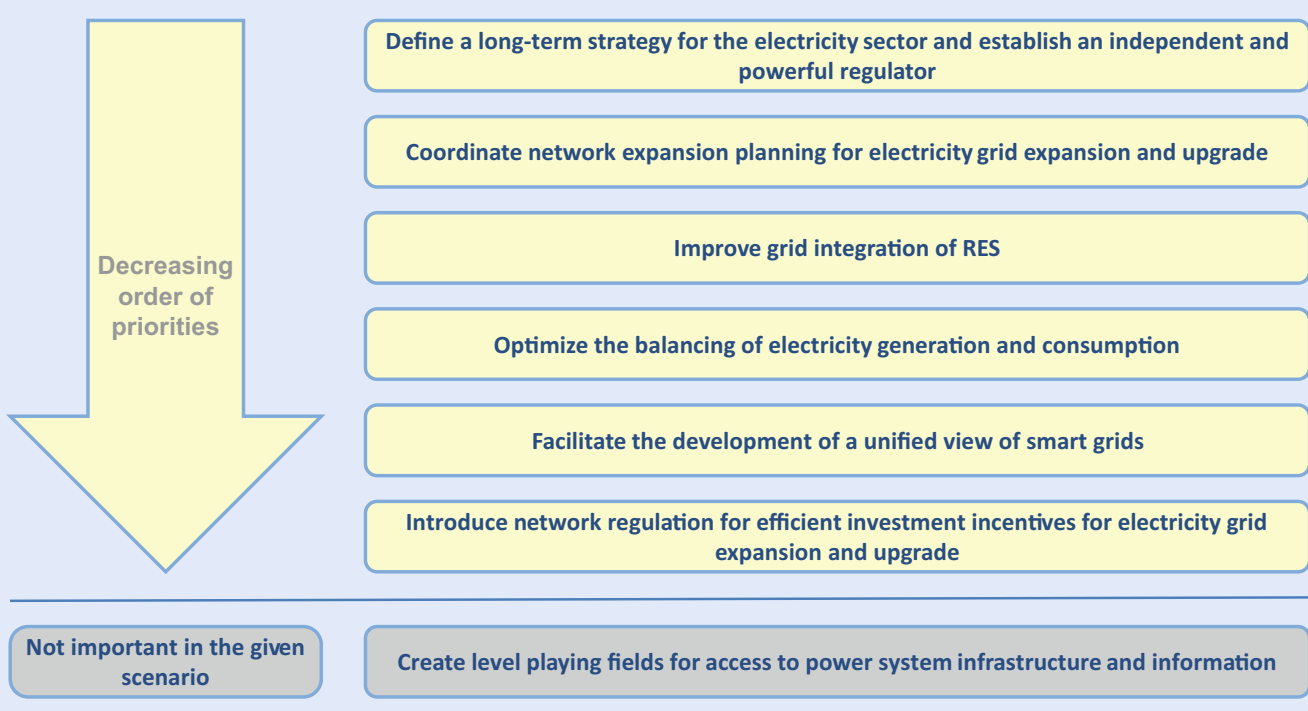

- Fig. 6.2 Priority of recommendations in the Reliability/Security of Supply scenario

- A clearly defined long-term strategy for electricity system development has to be specified by the government. The strategy should include long-term government targets with regard to the development of generation capacities of different generation technologies, and targets for energy efficiency. Such a strategy is a prerequisite for coordinated expansion of the electric power system as well as smart grid development. It might also serve as a point of reference for related government policies.

- The centralization of all regulatory aspects in one independent and powerful regulatory institution is of critical importance to increase the effectiveness of regulation. The main tasks of the regulator are coordination of network expansion planning and network regulation. In the Reliability/Security of Supply scenario, the regulator should specifically monitor power disruptions and outage times on national and local levels to provide an overview of the quality of electricity supply. Much like in Germany, grid operators should be obliged to regularly report data on power disruptions and outage times to the regulator (see $>$ Sect. 4.4.4 for related regulation in Germany). Pertinent laws (for example, a general energy law) should empower the Chinese regulator to effectively monitor power disruptions and outage times.

Coordinate network expansion planning for electricity grid expansion and upgrade Network expansion planning has a high priority in this scenario because the electric power grid is the backbone of the power system. Coordinated and efficient network expansion is the basis of a power infrastructure that can be adapted to consumer needs in a timely manner. This maximizes energy availability as the reliability of the future energy system essentially depends on the grid and generation capacities being matched to the consumption side.

Within this scenario, the main measures of the recommendation to Coordinate network expansion planning for electricity grid expansion and upgrade are: 
- In the short term, the main task to be accomplished for successful network expansion planning is to improve coordination between power system stakeholders and to define a common procedure for network expansion planning. Specifically, it is beneficial to coordinate network expansion planning and the construction of new generation capacities. The Future-oriented Energy Grids Platform can serve as a role model to encourage the establishment of a similar grid platform in China.

- In the medium term, stakeholders involved in the grid planning process have to elaborate a network development plan that will be mandatory for TSO and DSO under supervision of the regulator.

The measures to be implemented within the recommendation to Define a long-term strategy for the electricity sector and establish an independent and powerful regulator should precede the measures within the recommendation to Coordinate network expansion planning for electricity grid expansion and upgrade: a government strategy for the electric power system including long-term targets for generation capacities is a prerequisite for the network development process. An independent and powerful regulatory institution is supposed to supervise the network development process.

Improve grid integration of RES Increasing RES capacities contribute to supply the quickly increasing demand for electric power. Therefore, they support reliability and security of supply if the technical integration of RES is clearly defined. However, RES that have not been properly integrated may even endanger security of supply and stability.

Within this scenario, the main measures of the recommendation to Improve grid integration of RES are:

- In the short term, grid connection points must be defined for all kinds of RES at all grid levels.

- Defining a binding network code for grid connection that specifies responsibilities of grid operators as well as power generators is also a task in short term.

- RES generation capacities are needed in order to maximize available electricity and thereby reduce load curtailments. However, for grid stability and security of supply to be ensured, RES generation has to be curtailed under specific conditions. Therefore, procedures and documentation for curtailment of RES and the requirements in terms of IT support must be specified in the medium term.

Even if the recommendation to Improve grid integration of RES has high priority, measures to be implemented within several other recommendations serve as an input and should be implemented early: along with the need for an independent and powerful regulator to supervise curtailment procedures and define network codes, the clear specification of targeted RES generation capacities and well-defined network development procedures for network enhancement are preconditions for more effective RES integration. Also, a unified view of smart grids and technical standards contribute to a more effective integration of RES [7].

Optimize the balancing of electricity generation and consumption When electricity consumption increases so quickly that the growth of generation capacities can hardly follow, balancing and especially peak shaving are measures that support security of supply. Grid operation with higher safety margins eases grid control. Therefore, reduced peak loads resulting from peak shaving help to avoid system instabilities. Coordination of electricity generation and consumption is therefore of considerable priority in the scenario of reliability and security of supply. This includes load management, time-of-use pricing, and smart meter rollout.

Within this scenario, the main measures of the recommendation to Optimize the balancing of electricity generation and consumption are:

- In the short term, the main aspect is the further refinement of peak shaving mechanisms and technologies for system stabilization with focus on industrial and commercial consumers. One interesting option in this context is to tender the disconnectable loads on an internet platform (see Sect. 4.4.5 for related regulation in Germany).

- As already planned by the Chinese government, the time-of-use pricing system has to be 
refined (sufficient price differences between peak and off- peak prices for all categories of consumers) in the short term as well. A more sophisticated time-of-use pricing would incentivize investments in balancing mechanisms and technologies - for instance DSM, SSM, and energy storage.

An additional interesting measure originally not included in the recommendation to Optimize the balancing of electricity generation and consumption is locational pricing that can be used to signal network congestion. Locational pricing refers to power prices which are calculated for a number of locations - called nodes - on the transmission grid. Each node represents a physical location where power is injected by generators or withdrawn by loads [8]. Locational pricing reduces network congestion and may also set incentives for network expansion in regions with many network congestions [9]. It is an option for the long term and might become necessary in future in order to efficiently integrate the projected RES generation capacities. The regulator is supposed to supervise such locational pricing by regulating network charges with a clear scheme for the interaction between network operators and market players.

The discussion reveals that measures implemented within the recommendations to Define a long-term strategy for the electricity sector and establish an independent and powerful regulator and to Improve grid integration of RES serve as an input for the recommendation to Optimize the balancing of electricity generation and consumption.

Facilitate the development of a unified view of smart grids A technical reference architecture helps better understand smart grids and allows holistic analyses of security aspects relating to their implementation. Interoperability minimizes individual integration efforts and reduces the probability of interface failures. Such a reference architecture will therefore increase reliability and security of supply.

Within this scenario, the main measures of the recommendation to Facilitate the development of a unified view of smart grids are:

- In the short term, the main aspect is the creation and adoption of an organizational arrangement to model smart grid reference architectures and to coordinate smart grid standardization.

- Defining effective and efficient standardization processes (e.g. based on smart grid use cases) as well as coordinating the work of SDO to establish effective and efficient standardization processes are two further important aspects that should take place in the same time frame.

- In the medium term, a set of consistent smart grid standards should be created using the technical reference architecture.

Introduce network regulation for efficient investment incentives for electricity grid expansion and upgrade In the scenario focusing on reliability and security of supply, network regulation is somewhat less important but not irrelevant. It contributes indirectly to this scenario, as it sets economic incentives for the build-up and maintenance of the grid infrastructure. Incentives for efficient network investments and investments in R\&D and innovation are important in the context of the measures within this recommendation. These incentives, by contributing to the introduction of smart grid technologies, will directly ensure security of supply and reduce costs.

Within this scenario, the main measures of the recommendation to Introduce network regulation for efficient investment incentives for electricity grid expansion and upgrade are:

- In the short term, the use of incentive instruments like rate-of-return adder or innovation bonus should be assessed for application in China.

- A decision about the use of disaggregated regulation (i.e. regulating only the monopolistic bottlenecks) should be made in the short to medium term. If applicable, disaggregated regulation should be implemented in the medium term.

- Also in the medium term, incentives should be defined for investment in innovation and smart applications. In this context, the network development plan should be aligned and critical network connections for system stability which necessitate stronger investment incentives should be identified. At this point in time, a rate-ofreturn adder can be especially beneficial. 


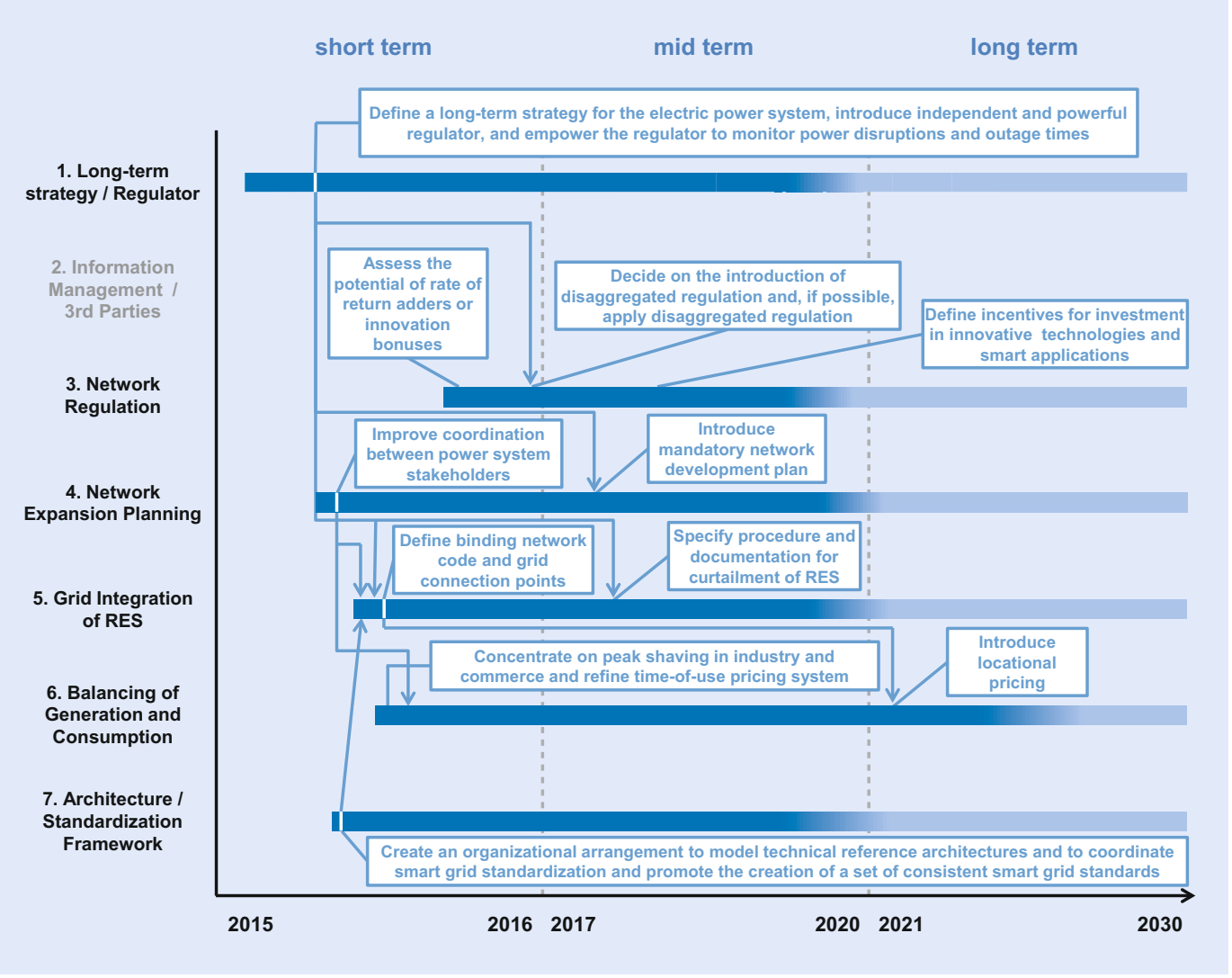

Fig. 6.3 Starting points, timeslots of main activities, and dependencies between main aspects of recommendations in the scenario focusing on reliability and security of supply

As an independent and powerful regulator is a prerequisite for network regulation, the recommendation to Define a long-term strategy for the electricity sector and establish an independent and powerful regulator should be realized before network regulation is focused upon.

Create level playing fields for access to power system infrastructure and information As new market actors are necessary more for innovation than for stability of the energy supply, the measures proposed in the recommendation to Create level playing fields for access to power system infrastructure and information are not considered as important in this scenario with a focus on reliability and security of supply.

Sequence of implementation In the light of the interdependencies described above, the following implementation sequence is proposed in the scenario focusing on reliability/security of supply:

- Define a long-term strategy for the electricity sector and establish an independent and powerful regulator.

- Coordinate network expansion planning for electricity grid expansion and upgrade.

- Facilitate the development of a unified view of smart grids.

- Improve grid integration of RES.

- Optimize coordination of electricity generation and consumption.

- Introduce network regulation for efficient investment incentives for electricity grid expansion and upgrade.

Figure 6.3 depicts the points in time for undertaking the main activities and shows dependencies 


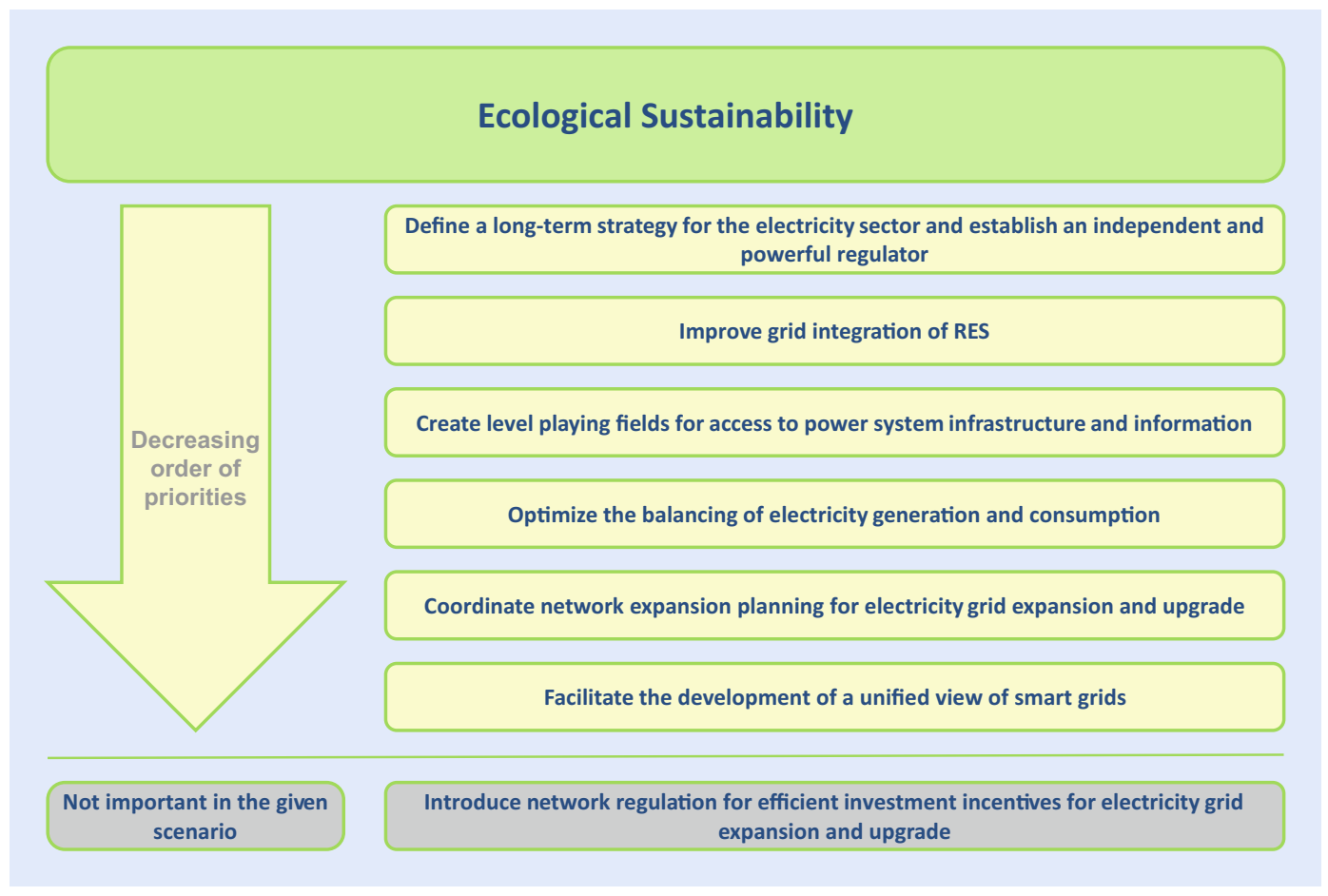

Fig. 6.4 Priority of recommendations in the Ecological Sustainability scenario

between the key aspects of recommendations for the scenario focusing on reliability and security of supply. Each recommendation is represented by a bar partitioned into timeframes from left to right. The starting point of each bar depicts the proposed beginning of work on each recommendation. Recommendations that are unimportant to the scenario are shown in gray. Proposed time points for the main measures are represented by the opaque blue regions on each bar. At the end of each bar, color gradients have been used to show that work on the recommendations continues even after the main aspects have been taken care of. The main measures are shown as boxes. The proposed starting time of each measure is shown by a diagonal line connecting the box to the bar. Dependencies between measures are shown by arrows while preconditions are marked with a white line crossing a bar at the beginning of the arrow. The end of the arrow points to the dependent measure.

\subsubsection{Ecological Sustainability scenario}

An introductory overview of the overall prioritization with respect to this scenario is presented in

- Fig. 6.4. It can be seen that the recommendations Define a long-term strategy for the electricity sector and establish an independent and powerful regulator, Improve grid integration of RES, and Create level playing fields for access to power system infrastructure and information have a high priority in the Ecological Sustainability scenario. Again, remember that these priorities do not necessarily correspond to the proposed implementation sequence because the interdependencies within the given scenario have to be analyzed before.

Define a long-term strategy for the electricity sector and establish an independent and powerful regulator As in the previous scenario, the commitment to a long-term strategy and the creation of an independent and powerful regulator has the highest priority in the scenario for ecological sus- 
tainability because a government-defined long-term strategy for the future electricity sector including specific RES expansion targets is a prerequisite for effective and efficient expansion of RES generation capacities.

Within this scenario, the main measures of the recommendation to Define a long-term strategy for the electricity sector and establish an independent and powerful regulator are:

- Effective regulation is required to ensure RES integration. Thus, the centralization of regulatory aspects in one independent and powerful regulatory institution should be addressed in the short term. Responsibilities for grid integration of RES and for power system information management should be assigned in this context.

- Official government targets for RES expansion are currently defined only until 2015. Within the forthcoming Five-Year Plan, the planning period will be extended to 2020. Defining a long-term government strategy concerning energy mix (including RES share), and energy efficiency indicators beyond 2020 is consequently a task for the medium term.

Improve grid integration of RES Timely connection and low curtailment of RES will speed up the reduction of greenhouse gas emissions as well as emissions of local and regional air pollutants. RES therefore need to be integrated into the grid with a very high priority and all of the following tasks need to be implemented in the short term:

- Proper definitions of grid connection points are necessary for all kinds of RES on all grid levels.

- A binding network code specifying responsibilities of both grid operators and power generators needs to be defined, as investors in RES need clear time limits for grid connection of RES in order to deliver generated electricity to the market at the right time. Grid operators should have to bear liability for grid connection of RES - resulting in a shallow cost approach for interconnection, whereby grid operators have both the responsibility and the opportunity for efficient overall grid development.
- Detailed procedures and documentation for curtailment of RES must be specified, and the requirements for IT support of these procedures must be clear. The implementation of this measure can be seen as a technical and economic framework to encourage investments into RES.

Measures related to the recommendation to Define a long-term strategy for the electricity sector and establish an independent and powerful regulator positively impact the effectiveness of the recommendation to Improve grid integration of RES: credible long-term government targets for RES generation capacities reduce risks related to investments in RES units. Another prerequisite is a powerful and independent regulator to supervise curtailment procedures and to define network codes.

\section{Create level playing fields for access to power sys-} tem infrastructure and information In principle, new market actors in China can already invest in RES. However, they need to be integrated into electricity sector governance more effectively in order to ensure efficient integration of RES. Non-incumbent market actors will invest in RES generation capacities only if the necessary information for competition and market access is available to them. Methods for the integration of the innovation potential of third parties have to be institutionalized and non-discriminatory information sharing has to be guaranteed.

Within this scenario, the main measures of the recommendation to Create level playing fields for access to power system infrastructure and information are:

- In the short term, the main measure of this recommendation is the definition of minimum necessary requirements for power system information management. Note that this task is related to the recommendation to Define a long-term strategy for the electricity sector and establish an independent and powerful regulator in - Fig. 6.5 due to the pivotal role of the government in this context.

- In the medium term, the role of new market actors in smart grids should be defined and how these actors can be integrated should be clarified. 
- An institution for power system information management should be specified, and this institution should set up a platform. It should then organize access to and exchange of information between all eligible parties.

Before the role of new market actors in smart grids can be defined, a long-term strategy for the electric power sector should be developed. Therefore, the recommendation to Define a long-term strategy for the electricity sector and establish an independent and powerful regulator should precede the recommendation to Create level playing fields for access to power system infrastructure and information. An architecture and standardization framework encourages the development of a unified smart grid model containing an overview of relevant use cases and business processes. Such a model, representing a form of Chinese SGAM, significantly eases the organization of power system information management. The platform for information management should therefore be implemented after the measures relating to the recommendation to Facilitate the development of a unified view of smart grids have been applied.

Optimize the balancing of electricity generation and consumption When consumption is not coordinated with generation, significant curtailments of RES generation are necessary to ensure grid stability. This reduces the attractiveness of investments in RES generation capacities. The usage of VPP, microgrids, or energy storage units facilitates the integration of distributed RES.

Within the scenario, the main measures of the recommendation to Optimize the balancing of electricity generation and consumption are:

- The first task in coordinating electricity generation and consumption is to promote VPP, microgrids, and energy storage technology for local integration in the short term by increasing $\mathrm{R} \& \mathrm{D}$ funding in this area.

- The time-of-use pricing system, which depends on a successful smart meter rollout, also has to be refined (sufficient price differences between peak and off peak prices for all categories of consumers) in the short term. This measure is already planned by the Chinese government. The first focus should be set on industrial and commercial consumers and then on residential consumers.

- Another interesting measure, originally not included in the recommendation to Optimize the balancing of electricity generation and consumption, is locational pricing. It might be interesting in the long term and contribute to RES integration (see $>$ Sect. 6.3.1 for a brief description of locational pricing).

Coordinate network expansion planning for electricity grid expansion and upgrade Network development is necessary to connect new consumers and generators to the network, including generation units belonging to third parties. The network development plan allows planning of RES installation and thereby increases investments in RES. As a consequence, network expansion planning is relevant in this scenario. The main measures of the recommendation to Coordinate network expansion planning for electricity grid expansion and upgrade should be taken in the short term:

- Increased coordination between established stakeholders and new market actors should be promoted by the government.

- A coordinated and mandatory network development plan including the aspect of RES integration should be established.

Based on experiences in Germany, an independent and powerful regulator is the best-suited organization for organizing and implementing these measures. Therefore, the recommendation to Define a long-term strategy for the electricity sector and establish an independent and powerful regulator should be implemented before the recommendation to Coordinate network expansion planning for electricity grid expansion and upgrade.

Facilitate the development of a unified view of smart grids As mentioned above, distributed energy resources are important and in this scenario their number is expected to rise. This implies an increase in system scale and complexity and calls for a smart grid architecture and standardization framework (i. e. an organizational arrangement to coordinate and promote smart grid standardization 


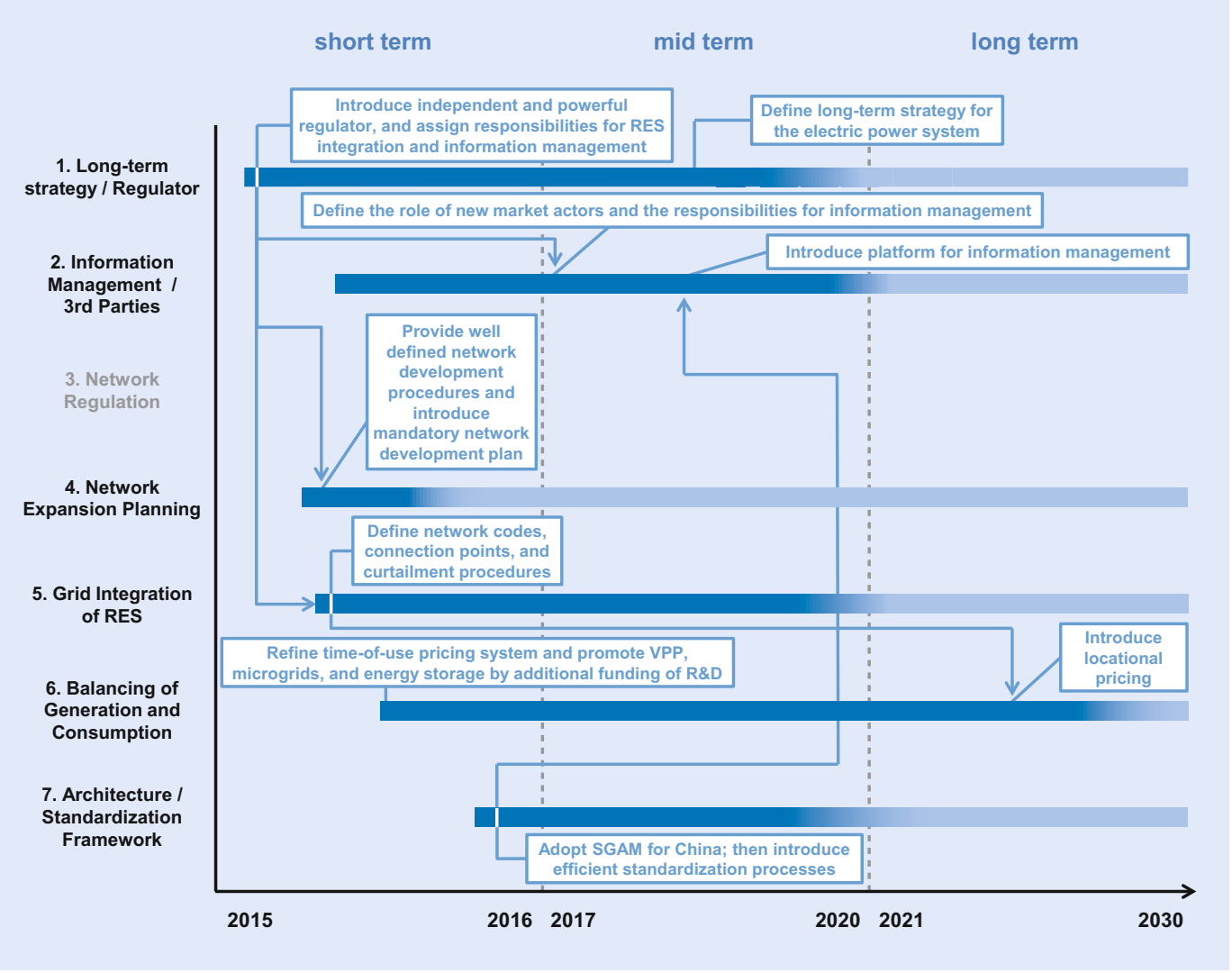

Fig. 6.5 Starting points, timeslots of main activities, and dependencies between main aspects of recommendations in the scenario focusing on ecological sustainability

and the development of a reference architecture). A unified view of smart grids should exist to elicit the resulting requirements and to compare different architectural solutions. The smart grid architecture and standardization framework should focus on interoperability between actors and systems, which is a key aspect in the context of decentralized system architectures. The high number of interfaces between systems implies the need for security analyses, which in turn requires solid models of systems architectures [7]. Clear structures and processes should be defined in order to coordinate the work of SDO.

Within this scenario, the main measures of the recommendation to Facilitate the development of a unified view of smart grids are:

- In the short term, an organizational arrangement to coordinate and promote smart grid standardization should be created and the development of a technical reference architecture framework should be assigned.

- In the medium term, effective and efficient standardization processes (e.g. based on a collection of smart grid use cases) should be established.

Introduce network regulation for efficient investment incentives for electricity grid expansion and upgrade For ecological sustainability, an electric power grid does not necessarily have to be developed at lowest costs. As network regulation focuses on efficient network investments, it is not an important regulation measure for this scenario.

Sequence of implementation • Figure 6.5 shows the timeframes of the main activities and interde- 


\section{Affordability/Competition}

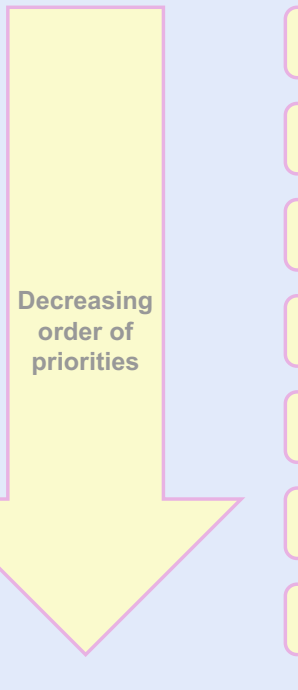

Create level playing fields for access to power system infrastructure and information

Coordinate network expansion planning for electricity grid expansion and upgrade

Define a long-term strategy for the electricity sector and establish an independent and powerful regulator

Introduce network regulation for efficient investment incentives for electricity grid expansion and upgrade

Facilitate the development of a unified view of smart grids

Improve grid integration of RES

Optimize the balancing of electricity generation and consumption

- Fig. 6.6 Priority of recommendations in the Affordability/Competition scenario

pendencies involved in this scenario. For a detailed description of the layout of the visualization, please check back to the previous section. In the light of interdependencies described above, the following implementation sequence is proposed in the scenario focusing on ecological sustainability:

- Define a long-term strategy for the electricity sector and establish an independent and powerful regulator.

- Improve grid integration of RES.

- Coordinate network expansion planning for electricity grid expansion and upgrade.

- Create level playing fields for access to power system infrastructure and information.

- Optimize the balancing of electricity generation and consumption.

- Facilitate the development of a unified view of smart grids.

\subsubsection{Affordability/Competition scenario}

An introductory overview of the overall prioritization with respect to this scenario is presented in - Fig. 6.6. It can be seen that the recommendations Create level playing fields for access to power system infrastructure and information, Coordinate network expansion planning, and Define a long-term strategy for the electricity sector and establish an independent and powerful regulator have a high priority in the Affordability/Competition scenario.

Create level playing fields for access to power system infrastructure and information In the scenario focusing on competition and innovation, two very important aspects are power system information management and the inclusion of new market actors. As non-incumbent market actors are main drivers for competition and innovation, non-discriminatory access to relevant information is crucial for them to encourage investments in the electric power system. China's smart grid development is dominated by SGCC and 
CSG, whereas the ICT industry hardly participates in strategic smart grid developments. It would be highly beneficial to change this situation with regard to the non-involvement of the ICT sector and to create business opportunities for new market actors.

Within this scenario, the main measures of the recommendation to Create level playing fields for access to power system infrastructure and information are:

- In the short term, the definition of the role of new market actors in smart grids and how these actors can be integrated is one of the main aspects.

- Defining minimum requirements for a data platform to organize access to and exchange of power system information between all eligible parties has to be accomplished in the short term.

- Also, the establishment of such a data platform is another task to be accomplished in this timeframe.

A common understanding of the structural aspects of smart grids would encourage the identification and description of the roles of new market actors as well as their need for information and interfaces both on the business and technical level. An institutionalized smart grid architecture and standardization framework might therefore serve as an intercompany interaction mechanism to strengthen the role of third parties even beyond standardization.

Coordinate network expansion planning for electricity grid expansion and upgrade The recommendation to Coordinate network expansion planning for electricity grid expansion and upgrade proposes that a stakeholder platform for network development should be established. This platform would mean more involvement of all market actors. All measures associated to this recommendation should be implemented in the short term:

- Coordination between established power system stakeholders and new market actors has to be strengthened. For this purpose, a stakeholder platform similar to the Future-oriented Energy Grids Platform in Germany could be established and managed by a government institution.
- Common procedures for network expansion planning should be defined. A network development plan should be elaborated within a coordinated process and shared responsibilities and made mandatory afterwards.

- Responsibilities for network planning should be identified, thereby clarifying which new market actors should participate in this process. This task shall be accomplished by a government organization, but it can be supported by grid operators.

Before the start of network expansion planning including new market actors, the role of new market actors in China's electric power system must be defined. Therefore, the recommendation to Create level playing fields for access to power system infrastructure and information should precede the recommendation to Coordinate network expansion planning for electricity grid expansion and upgrade.

Define a long-term strategy for the electricity sector and establish an independent and powerful regulator As government agencies have to supervise and guide grid operation, an effective definition of the government's roles and responsibilities is important. This ensures favorable conditions for the development and rollout of innovations.

Within this scenario, the main measures of the recommendation to Define a long-term strategy for the electricity sector and establish an independent and powerful regulator are:

- If the responsibilities for network planning have not already been defined within the previous recommendation, the government has to define these responsibilities in the short term.

- An independent and powerful regulator should be established in the medium term.

- A long-term strategy for the future electric power sector beyond 2020 must be defined in the same time frame.

Introduce network regulation for efficient investment incentives for electricity grid expansion and upgrade In the scenario focusing on affordability and competition, disaggregated regulation should ensure that only monopolistic bottlenecks (transmission and distribution grids) are regulated. Con- 
sequently, competition can evolve in all other stages of the supply chain and incentives are created for a cost-efficient build-up of the grid and for technical innovations.

Within this scenario, the main measures of the recommendation to Introduce network regulation for efficient investment incentives for electricity grid expansion and upgrade within this scenario are:

- Network charges offering efficient investment incentives in smart grids have to be introduced in the medium term.

- After network charges have been fixed, disaggregated regulation can be introduced. Due to the high impact of disaggregated regulation on competition in the retail sector, this measure should also be implemented in the medium term.

Before network regulation can be established, the responsibility for network regulation has to be defined and an independent and powerful regulator is necessary.

Facilitate the development of a unified view of smart grids As mentioned above, a smart grid architecture and standardization framework (i.e. an organizational arrangement to coordinate smart grid standardization and the development of a reference architecture) can be institutionalized as an inter-company interaction mechanism to strengthen the role of new market actors. In addition, it ensures interoperability between solutions coming from different vendors, prevents vendor lock-in and makes Chinese smart grid technologies applicable in international markets as well as vice versa [10], [11]. Furthermore, the framework supports the standardization and engineering process and thereby the diffusion of knowledge.

Within this scenario the main measures of the recommendation to Facilitate the development of a unified view of smart grids are:

- In the short term, the smart grid architecture and standardization framework has to be created or adopted to enable modeling of smart grid solution architectures.

- Coordinating the work of organizations involved in the development of standards needs clear structures and incentives for new market actors to participate in the process. The defini- tion of these structures and incentives is also a short-term task.

- In addition, standardization processes need to be coordinated with international standardization in the short term in order to establish effective and efficient standardization processes (e. g. based on a collection of smart grid use cases) in the medium term.

Improve grid integration of RES Grid integration of RES is important in this scenario. The stricter the conditions for grid connection of RES are formulated, the less smaller power generation companies are dependent on the grid operators. Rules favorable for the integration of RES attract new investors, i.e. third parties that need both stable investment and technical conditions. The following measures should therefore be implemented in the short term in order to allow competition in the field of RES generation:

- Grid connection points need proper definition for all kinds of RES on all grid levels.

- A binding network code for grid connection specifying the respective responsibilities of grid operators and power generators needs to be specified. In this context, grid operators should bear liability for grid connection of RES.

- A detailed procedure and documentation for curtailment of RES including, for example, document exchange and transparency rules must be defined. Additionally, the requirements for IT support for these procedures need to be specified.

Well-defined network development procedures for network enhancement involving RES will positively impact the effectiveness of these measures. The recommendation to Coordinate network expansion planning for electricity grid expansion and upgrade should therefore start before the recommendation to Improve grid integration of RES.

Optimize the balancing of electricity generation and consumption The recommendation with the lowest priority in this scenario is the balancing of electricity generation and consumption, which is relevant for integrating prosumers in the electric power system. Balancing of electricity generation and consumption includes load management, time- 


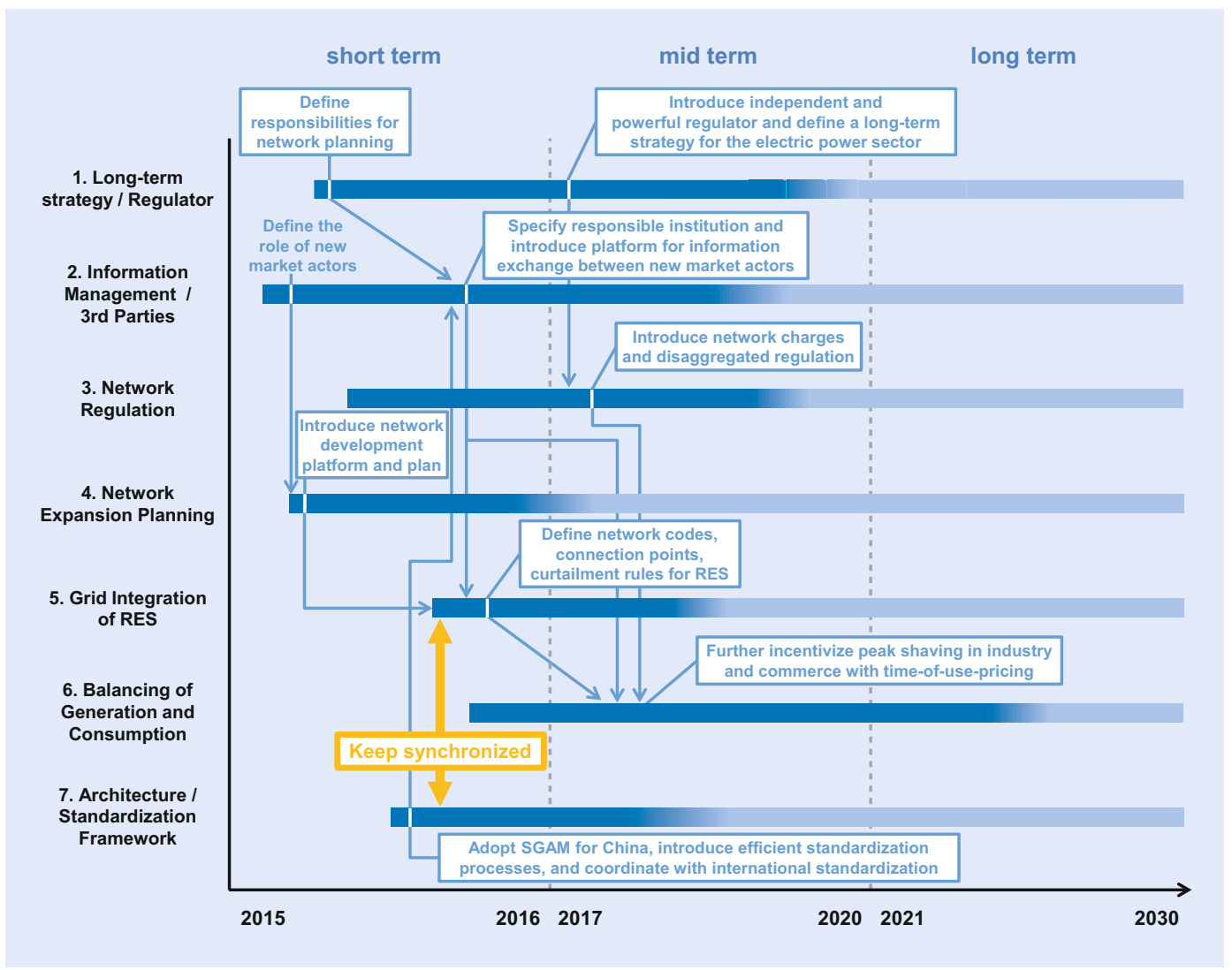

- Fig. 6.7 Starting points, timeslots of main activities, and dependencies between main aspects of recommendations in the scenario focusing on competition and innovation

of-use pricing, smart meter rollout, and the promotion of VPP, microgrids, and energy storage by reducing barriers to their implementation.

Within this scenario, the main measures of the recommendation to Optimize the balancing of electricity generation and consumption are:

- In the medium term, coordination between electricity generation and consumption can be optimized by peak shaving and a further refinement of time-of-use pricing. Such a time-of-use based framework for pricing with sufficient price differences will incentivize the usage of balancing mechanisms and technologies such as DSM, SSM, and energy storage.

Balancing of electricity generation and consumption potentially depends on the grid integration of RES. However, power system information manage- ment with smart meter infrastructure and information interchange between all parties as well as an independent and powerful regulator for regulation of network charges are also necessary.

Sequence of Implementation • Figure 6.7 shows the timeframes of main activities and interdependencies. In the light of the interdependencies described above, the following implementation sequence is proposed in the scenario focusing on ecological sustainability:

- Create level playing fields for access to power system infrastructure and information.

- Coordinate network expansion planning for electricity grid expansion and upgrade.

- Define a long-term strategy for the electricity sector and establish an independent and powerful regulator. 
- Introduce network regulation for efficient investment incentives for electricity grid expansion and upgrade.

- Facilitate the development of a unified view of smart grids.

- Improve grid integration of RES.

- Optimize coordination of electricity generation and consumption.

\subsection{Discussion of the three roadmaps}

In addition to the specific timeline of each scenario, the three different roadmaps presented above give policy makers the following general hints:

- The priority and relevance of each recommendation can be assessed on the basis of the underlying scenario. For example, it can be seen that the measures of the recommendation to Create level playing fields for access to power system infrastructure and information have the highest priority if the government focuses on fostering competition and innovation but are virtually irrelevant if the government focuses only on reliability issues.

- Comparing the proposed implementation sequences in all three scenarios shows which recommendations have high priorities in all three scenarios. Such recommendations can be considered as political imperatives and should be implemented irrespective of the underlying policy goals of the Chinese government.

Figure 6.8 summarizes the proposed implementation sequences for all three scenarios. Those recommendations to be implemented in the beginning are presented in the left. For example, the Ecological Sustainability scenario starts with Define a long-term strategy for the electricity sector and establish an independent and powerful regulator, followed by Improve grid integration of RES and then by the remaining recommendations.

Comparing the implementation sequences in all three scenarios reveals that there are two recommended approaches with the highest overall priority. The measures subsumed within these recom- mendations shall be implemented independently of the underlying scenario:

- Define a long-term strategy for the electricity sector and establish an independent and powerful regulator, and

- Coordinate network expansion planning for electricity grid expansion and upgrade.

Three of the remaining five recommendations are relevant in each scenario, though with a lower priority:

- The Improvement of the grid integration of RES has a very high priority under the Ecological Sustainability scenario and is also important for the Reliability/Security of Supply scenario. It is somewhat less important in the Affordability/Competition scenario.

- The Facilitation of the development of a unified view of smart grids is especially important with respect to the Reliability/Security of Supply scenario. It is somewhat less important with regard to the Affordability/Competition and Ecological Sustainability scenarios.

- The Optimization of the balancing of electricity generation and consumption is particularly relevant for the Reliability/Security of Supply scenario. The recommendation has a lower relevance in the Ecological Sustainability scenario and is ranked last in the Affordability/Competition scenario.

The two remaining recommendations are not relevant in every scenario. Rather, they contribute to single energy policy goals. In particular, both of these recommendations are essential for migrating towards smart grids focusing on affordability and competition:

- The Creation of level playing fields for access to power system infrastructure and information is the first recommendation that should be implemented if the government strives to develop smart grids focusing on affordability and competition. It is also important if the government chooses to focus on ecological sustainability.

- The Introduction of network regulation for electricity grid expansion and upgrade is at position four in the implementation sequence 


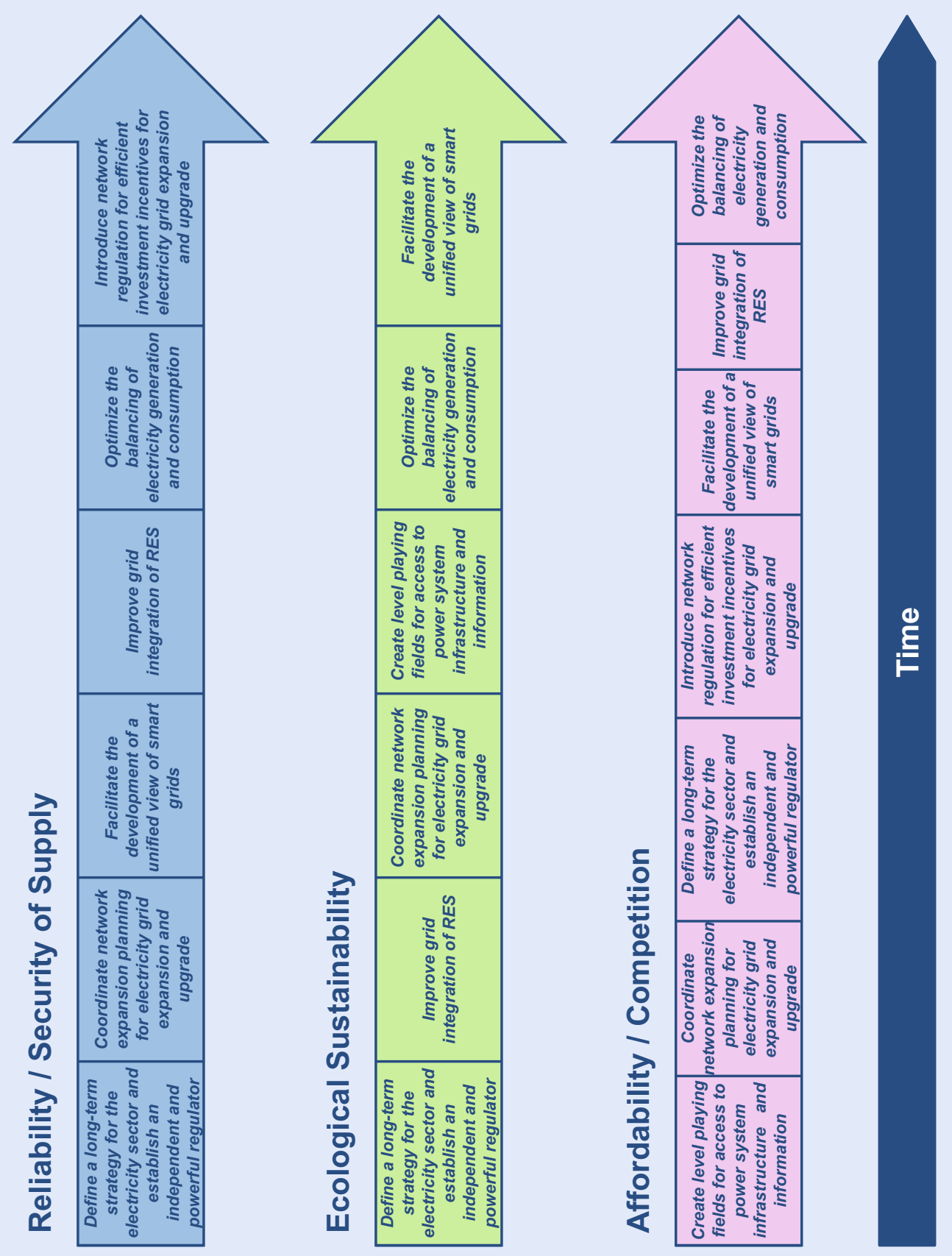

- Fig. 6.8 Overview of proposed implementation sequences for all three scenarios 
of the Affordability/Competition scenario. It is also relevant, though at a later stage, in the Reliability/Security of Supply scenario.

\section{References}

1 State Council of the People's Republic of China, "12th FiveYear Plan for Energy Development," Guofa, Beijing, 2013.

2 State Council of the People's Republic of China, "Opinions on Accelerating the Development of Environmental Industry," Beijing, 2013.

3 National Energy Administration (NEA), "Key Information at a Glance - China 12th Five-Year Plan for Renewable Energy Development," China National Renewable Energy Center (CNREC), Beijing, 2012.

4 State Council Information Office of the People's Republic of China (SCIO), “China's Energy Policy 2012," SCIO, Beijing, 2012.

5 International Energy Agency (IEA), “Understanding China's 12th Five-Year Energy Plan," IEA, Paris, 2013.

6 G. Tong, "Status Quo of the Smart Grid Development in China and Its Driving Forces," National Energy Administration (NEA), Oldenburg, 2013.

7 Bundesverband der Energie- und Wasserwirtschaft (BDEW), "BDEW-Roadmap: Realistische Ziele zur Umsetzung von Smart Grids in Deutschland," BDEW, Berlin, 2013.

8 D. Philliips, "Nodal Pricing Basics," [Online]. Available: http://www.iemo.com/imoweb/pubs/consult/mep/LMP_ NodalBasics_2004jan14.pdf. [Accessed February 21, 2014].

9 S. Stoft, Power System Economics, Piscataway:The Institute of Electrical and Electronics Engineers, 2002.

10 B. Quélin, T. Abdessemed, J.-P. Bonardi and R. Durand, "Standardization of Network Technologies: Market Processes or the Result of Inter-Firm Co-Operation?," Journal of Economic Surveys, vol. 15, no. 4, pp. 543-569, 2001.

11 G. Tassey, "Standardization in Technology-Based Markets," Research Policy, vol. 29, no. 4-5, pp. 587-602, 2000.

Open Access This chapter is distributed under the terms of the Creative Commons Attribution Noncommercial License, which permits any noncommercial use, distribution, and reproduction in any medium, provided the original author(s) and source are credited. 